\title{
Experimental Ophthalmology
}

National Cancer Institute

\section{Source}

National Cancer Institute. Experimental Ophthalmology. NCl Thesaurus. Code C18780.

Basic research in the field of ophthalmology. 